\title{
Erst Praxis, dann Theorie
}

Business Excellence ist integriert oder schon im Ansatz zum Scheitern verurteilt. Business Excellence ist ein viel gehörtes Schlagwort. Business Excellence will jeder. Wie aber kann Business Excellence gelingen? Gute Ansätze gibt es zuhauf. Eine überzeugende ganzheitliche Perspektive, ein integrierendes Gesamtbild ist jedoch noch zu liefern. Damit, wie eine erfolgreiche Integration von Business Excellence gelingen kann, befasst sich der vorliegende Text. Antworten, Erfolg versprechende wie erfolgreiche, kommen aus der Praxis. Ausgangspunkt der Überlegungen bildet eine praxeologische Reflexion der Change Managementund Organisationsentwicklungsarbeit im Sinne des Action Research und Systems Research (Klein 2016b; Edson und Klein 2016). Für das Thema Business Excellence wurden insbesondere die Erfahrungen aus der Begleitung von ChangeManagement-Prozessen im Energiegroßanlagenbau zur Referenz neuer Ideen und Entwicklungen ausgewertet.

Integration braucht Reflexion. Und Reflexion braucht eine leistungsfähige Theorie. Die Dynamik der Welt ist multikausal und interdependent. Damit konfrontiert kommt man um systemisch-kybernetische Ansätze nicht herum. Sie liefern Beobachtungsinstrumente, Modelle und Methoden, mittels derer sich Lern- und Entwicklungspfade beschreiben lassen, die im Total-Quality-Management (TQM) ihren Anfang nehmen und von der Operational Excellence der Produktion zur Project Excellence führen. Es wird weiter zu zeigen sein, dass für erfolgreiche Organisational Excellence in Produktion und Projekten ein ebenso exzellentes Widerlager in den managerialen Governance-Prozessen erforderlich ist. Unter Berücksichtigung der Zeit und des unumgänglichen Wandels wird dann aus dem Wechselspiel von Organisational Excellence und Change Excellence etwas Integriertes, das den Namen Business Excellence verdient, doch nur zum Preis neuer blinder Flecken, wie zum Beispiel im Bereich Sales. 